Itinéraires Itinéraires

Littérature, textes, cultures

\title{
Variation sur une typologie des corps dans l'imaginaire littéraire au Maroc
}

\section{Abdallah Lissigui}

\section{(2) OpenEdition}

\section{Journals}

Édition électronique

URL : http://journals.openedition.org/itineraires/1507

DOI : $10.4000 /$ itineraires. 1507

ISSN : 2427-920X

Éditeur

Pléiade

\section{Édition imprimée}

Date de publication : 1 novembre 2011

Pagination : 61-74

ISBN : 978-2-296-55720-8

ISSN : 2100-1340

\section{Référence électronique}

Abdallah Lissigui, « Variation sur une typologie des corps dans l'imaginaire littéraire au Maroc », Itinéraires [En ligne], 2011-3 | 2011, mis en ligne le 01 novembre 2011, consulté le 01 mai 2019. URL : http://journals.openedition.org/itineraires/1507 ; DOI : 10.4000/itineraires.1507

\section{(c) (i) $(9$}

Itinéraires est mis à disposition selon les termes de la licence Creative Commons Attribution - Pas d'Utilisation Commerciale - Pas de Modification 4.0 International. 


\title{
Variation sur une typologie des corps dans l'imaginaire littéraire au Maroc
}

\begin{abstract}
This article proposes to explore a typology of body which worries the postcolonial imaginary in Morocco. It is especially question of those of the woman, the homosexual and the father. The fourth is of inter-semiotic nature. These bodies contain an improper dimension, in break with a conventional and normative axiology. They also contain a major socio-cultural stake which reveals a set of fluctuations which the subject undergoes in the contemporary Moroccan society, torn between tradition and modernity, the fascination for the desire of the body and the dismay it generates when it becomes sharp, exalted and unbridled. This new position of the body refers to several characters in search of an idyllic human condition and an identity to re-build.
\end{abstract}

Keywords : body, postcolonial imaginary, axiology, Moroccan literature, identity Mots clés : corps, imaginaire postcolonial, axiologie, littérature marocaine, identité

\section{Introduction}

Ce travail vise à étudier certains éléments constitutifs de l'imaginaire du corps littéraire qui prédomine ${ }^{1}$ et préoccupe en permanence la fiction

1. Lorsque l'on parcourt la bibliographie afférente à la thématique du corps depuis son émergence jusque dans ses productions les plus récentes, l'on s'aperçoit facilement qu'elle demeure au cœur des préoccupations majeures de la littérature marocaine contemporaine. Sa réception critique est aussi au confluent d'une interdisciplinarité et d'une multitude d'approches qui la rendent de plus en plus heuristique et complexe. L'avènement du mot corps se dévoile d'emblée dans certains titres d'œuvres de fiction dont nous citons ici quelques exemples : Le Corps dérobé, de Houria Boussajra, Corps négatif de Mohamed Khaïr Eddine, Un corps et une ville de Zhor Gourram, Les Fruits du corps de Abdellatif Lâabi. Il n'en reste pas moins que c'est sur les plans de la diégèse et de l'écriture que l'imaginaire du corps semble renforcer son ubiquité chez une grande majorité d'écrivaines et d'écrivains de la première comme de la dernière génération. 
romanesque marocaine. Une fiction où le culte, la culture et la sculpture du corps continuent résolument à étendre leur emprise sur les écrivains, sur leur représentation de soi et de l'autre, souvent variable et controversée. Penser le corps littéraire dans le contexte postcolonial au Maroc nous semble être un acte complexe en raison notamment de l'hétérogénéité culturelle qui le distingue et de la crise dans le système des valeurs qui lui est associé. Notre hypothèse consiste à envisager le corps comme étant le vecteur essentiel de l'interprétation du texte et comme le principal générateur de la narration postcoloniale. Suivant l'imaginaire de cette narration, le corps se met constamment en quête de sa mémoire, de son être et de sa raison d'être. Dans ce contexte, point n'est besoin de s'apercevoir que la forme du corps qui habite cet imaginaire demeure fascinante et lancinante à la fois.

Nous nous proposons de montrer dans cet article comment les écrivains marocains se démarquent les uns des autres dans leur identification du corps, de sa représentation et de sa manière de paraître et d'être. Pour ce faire, nous passerons en revue une typologie de corps humains, suivant un dispositif composite et varié de poses et de positions. À partir de cette variation littéraire des corps et en l'absence d'un corps « normal », typique, il ressort que notre corpus se limitera à quatre types de corps qui seraient porteurs de plusieurs signes dont l'observation ne semble pas fléchir devant leurs détails inconvenants. Ils concernent particulièrement ceux de la femme, de l'homosexuel et du père. Le quatrième est de nature strictement intersémiotique. Tous ces corps renferment une dimension inconvenante, en rupture avec une conception normative, et augurent, en même temps, un enjeu socioculturel et axiologique majeur.

\section{Corps de femme et crise de l'être}

Parmi les œuvres de fiction que nous avons interrogées, celles des écrivaines surprend, d'emblée, par la hantise de la thématique du corps féminin qui persiste dans leur énonciation et dans leur pratique d'écriture. Cette préoccupation des auteurs à l'égard de leur propre corps procède principalement de la prise de conscience qu'elles éprouvent face aux enjeux afférents au statut du corps tant sur le plan culturel que social et idéologique. Si, en effet, le corps multiplie ses mises en scène dans l'imaginaire féminin, c'est qu'il est soumis aux diverses mutations que traverse la société marocaine contemporaine. Face à ces mutations, le corps de la femme se dote d'un caractère centrifuge à même de se dévoiler dans sa splendeur et sa misère, dans ses forces et ses faiblesses, dans son enchantement et son désenchantement. Cette profusion des corps en représentation dans les textes, devient le lieu d'une crise par où se font et se défont les valeurs de la modernité, ses exigences et ses contradictions. Chez de nombreuses romancières, en effet, la fictionalisation du corps féminin participe d'une pluralité de situations stéréotypées en rapport avec le vécu social et culturel environnant. Les héroïnes font souvent figure de jeunes 
femmes velléitaires, tantôt assujetties à la suprématie masculine, tantôt déchaînées jusqu'au bout contre l'ordre établi. Ce rapport de force les conduit souvent à se constituer en sujet conscient de sa position sociale, en un sujet de désir de soi-même et de l'autre, désireux de son corps et de son identité.

Ainsi, dans le roman Marrakech, lumière d'exil de Rajae Benchemsi, par exemple, la narratrice décrit le vécu corporel et social de l'héroïne, Bradia, sur un ton foncièrement pathétique. Contrariée et mutilée dans son âme et son corps, la vie de Bradia est imprégnée de signes de disgrâce qui révèlent un « je » souffrant et meurtrie par tant d'échecs et de déboires :

Le temps de la douleur avait achevé d'imprimer, dans le vide effroyable de sa voix, les vicissitudes de sa vie. La joie. L'amertume. La détresse ${ }^{2}$.

Les hommes avaient fait valoir la loi, ils n'avaient plus qu'à se retirer. Leur rôle était précis et circonscrit. Eux écrivaient la loi. Les femmes écrivaient la vie. De leurs rires. De leur perspicacité. De leur corps mais aussi de leur sang et de leur sagacité ${ }^{3}$.

Dans Femmes inachevées de Houria Boussejra comme dans Anissa captive de Fatiha Boucetta, les héroïnes sont également ruinées et suppliciées dans leurs corps blessés comme le montrent les deux extraits suivants :

Je n'étais qu'un semblant d'être humain, qui ne devait jamais rien entreprendre mais toujours subir. Le verbe se faisait violence dans l'univers glacé de mes désirs. J'avais abandonné le rêve, depuis mon éveil au monde [...]. Étrangère à moi-même, j'habitais un corps, j'avais une parole qui ne ressemblait à rien ${ }^{4}$.

La société, ce monstre sacro-saint sans l'approbation duquel personne ne peut faire un pas, pour lequel je suis arrivée là où je suis : malheureuse, mariée avec un homme que je n'ai jamais aimé et surtout avec lequel je ne m'entends pas. [...] Je suis toujours captive ${ }^{5}$.

Aussi, dans Le Concert des cloches de Souad Bahéchar, la jeune fille Warda, qui n'en pouvait plus de subir l'arrogance masculine, finit par soutenir que les hommes sont « tous des fruits véreux, juste bons pour une mauvaise confiture ${ }^{6} \gg$.

Ces premiers exemples semblent emblématiques de toute une génération d'œuvres issues de la littérature dite « féminine ». Au Maroc, l'avènement de cette littérature a pour enjeu essentiel d'opérer d'une manière

2. Rajae Benchemsi, Marrakech, lumière d'exil, Paris, Wespieser, 2006, p. 33-34.

3. Ibid., p. 48-49.

4. Houria Boussejra, «Aïcha », dans Femmes inachevées, Rabat, Marsam, 2002, p. 45-46.

5. Fatiha Boucetta, Anissa captive, Casablanca, Eddif, 1991, p. 297.

6. Souad Bahéchar, Le Concert des cloches, Casablanca, Le Fennec, 2005, p. 14. 
irréversible un éclatement des rôles et une inversion ${ }^{7}$ dans le rapport de force qui caractérise souvent le masculin et le féminin. Il s'agit manifestement d'une prise de position résolument engagée à s'indigner contre le poids de la censure et à briser le mutisme et le vide dans lesquels le corps féminin a été pour longtemps enfoui. Pour les écrivaines, cette aversion pour les hommes résulte de leur prise de conscience d'une réalité insoutenable et qu'il importe de dénuder et de dénoncer en même temps.

Un autre topos majeur où le corps féminin s'ingénie à se construire, dans l'écriture féminine, concerne la passion amoureuse. Dans plusieurs occurrences romanesques, chez Rajae Benchemsi, par exemple, le corps féminin s'instaure, non pas comme un objet de désir, mais comme un sujet désirant, sujet qui se réjouit à s'assumer dans un contact charnel et voluptueux. C'est le cas dans le passage suivant tiré de Fracture du désir :

Je regarde tantôt son visage tantôt le mien pour détecter ma part de mort et ma part d'érotisme. Je me mets à quatre pattes et hume la chaleur de son corps qui m'emplit d'une gloire sournoise et intrépide. [...] L'introversion est totale : je suis elle et elle est moi. Je parcours son corps de mes lèvres chaudes et ses flammes de feu brûlent mon âme égarée ${ }^{8}$.

La scène de la relation charnelle entre ces deux femmes, explicite et confidentielle à la fois, vise à rompre sans doute avec une doxa établie et à tourner en dérision une vision éthique et conventionnelle de la sexualité. Conquis et explorés dans presque tous ses plis et replis morphologiques extrêmes, le corps féminin, en raison de cette force éruptive qui l'habite, se fait et se défait suivant un double mouvement d'intériorisation et d'extériorisation, d'intimité et d'extimité. Devenu de plus en plus autonome, le sujet féminin n'hésite plus à « montrer » ce qui devrait être " caché $^{9}$ ", à représenter l'irreprésentable et à narrer l'inénarrable. Il s'agit de donner libre cours à une passion amoureuse sublime qui s'opposerait aux pratiques masculines jugées viriles et humiliantes.

7. Lors d'une scène de dialogue entre deux femmes, l'esclave et sa maîtresse dans Marrakech, lumière d'exil de Rajae Benchemsi, le corps est toujours sollicité pour servir un désir latent d'assujettir l'homme et de le dompter. Lisons ce passage : « Ne succombe jamais à l'amour. Jamais m'entends-tu? Apprends à le dompter. Comme un fauve. Une mer déchaînée. Un ouragan. Sois toujours prête en cas de vertige à le laisser échouer comme un vaisseau à la dérive. Les hommes sont violents avec les esclaves de l'amour» (p. 67-68).

8. Rajae Benchemsi, « Elle », dans Fracture du désir, Arles, Actes Sud, 1999, p. 120.

9. Selon Philippe Hamon, le corps qui est vu comme une « chose cachée et intime par excellence, sera donc l'un des lieux privilégiés de fixation, sous la forme d'une herméneutique, de ce fantasme réaliste. D'où une conception du corps comme composé de diverses enveloppes emboîtées et solidaires (la peau, le vêtement, la maison, l'espace social des rites et des rituels) et de ses diverses boîtes anatomiques (le cerveau, le ventre) un corps qui émet, produit, supporte, reçoit, inscrit et stocke images, signes, symptômes, indices, insignes et signaux de tout genres » (Imageries, littérature et images au XIX $X^{e}$ siècle, Paris, José Corti, p. 186). 
Dans d'autres occurrences, les personnages féminins se réconcilient intimement avec le secret de leur corps radieux, se rallient gracieusement avec les traits saillants de leur beauté et de leur féminité. Aussi se refusentils à toute forme d'inféodation et de servilité comme on peut le lire dans trois autres extraits successivement tirés de Cérémonie et de Marrakech, lumière d'exil.

Le corps de Aïcha qui est blanc et plein s'épanouissait dans la chaleur du bain, les aréoles de ses seins s'évasaient, de ses jambes closes et repliées s'échappait un sombre buisson, quand elle levait les bras, un duvet flottait à ses aisselles profondes ${ }^{10}$.

La plus grande crainte de Bradia était d'avoir à renoncer à sa personne [...]. Rien, pas même le lourd système patriarcal de sa nouvelle famille, ne l'avait réduite à vivre à l'ombre de ses désirs ${ }^{11}$.

Elle était allongée sur le ventre, continua Lalla Tata, face au miroir et s'était appuyée sur les coudes. Soutenant son visage de ses mains, elle se contemplait et se trouvait belle. [...] Elle avait suivi l'arrondi de ses épaules et l'avait trouvé parfait. Avait effleuré son cou du bout des doigts et dit à voix haute : «rien ni personne ne me détournera de mes désirs. Rien ${ }^{12}$.

À partir de ces exemples, il s'avère que le désir de ces héroïnes est de pouvoir persister à se tenir libre de bout en bout, en dépit d'un destin irrésistible et tragique. Leur voix, ne trouvant pas d'écho favorable devant une réalité hostile et menaçante, continue à frayer un chemin qui mène les protagonistes à vivre pleinement leur propre désir dans une relation introvertie, au travers d'un miroir révélateur d'un narcissisme inédit et complaisant.

Aux yeux de ces écrivaines, la dimension sensuelle du corps devient alors une alternative et un choix dans la construction de l'identité du sujet féminin. Car elle permet à la fois de conquérir une connaissance « jouissive » de soi et de contester en même temps le pouvoir masculin et l'effet de la discrimination qui en résulte. L'exhibition de la nudité ${ }^{13}$ féminine

10. Yasmine Chami-Kettani, Cérémonie, Arles, Actes Sud, p. 86.

11. Rajae Benchemsi, Marrakech, lumière d'exil, op. cit., p. 58.

12. Ibid., p. 138.

13. La couverture illustrée des œuvres de certaines romancières marocaines est de plus en plus ouverte sur une thématique du nu et de l'érotisation du corps féminin. C'est le cas, par exemple, de Fracture du désir de Rajae Benchemsi, déjà cité, de Demoiselles de Numidie de Mohamed Leftah (Paris, Édition la Différence, 2006), de Le Harem européen de Fatima Mernissi (Paris, Albin Michel, 2001) et de Une femme tout simplement de Baha Trabelsi, (Casablanca, Eddif, 1995). Au regard des différentes images couvrant les paratextes picturaux de ces œuvres, les signes du corps qui sont visibles à « l'œil nu » dévoilent une corporalité sombrant dans la décrépitude et le mal-être des personnages à l'œuvre dans ces textes. On comprend alors mieux que si le corps se laisse souvent plier aux signifiés culturels, il deviendra vite attentatoire aux signes de la convenance dès lors qu'il se dénude. La mise 
constitue ici une forme de dissidence et un signe annonciateur de la quête d'une nouvelle individualité dans un espace social qui confine souvent le corps dans une vision stéréotypée et réifiée. C'est pourquoi il est étonnant de remarquer que les narratrices recourent moins aux euphémismes pour lever le voile sur la nudité des héroïnes et sur leur récit érotique ${ }^{14}$. La focalisation sur cette beauté efféminée dote le corps érogène ${ }^{15}$ du sujet féminin d'un pouvoir irrépressible, pouvoir qui lui permet de se refuser à l'abandon et à la passivité. Ce nouveau mode de représentation du corps conduit les héroïnes à exalter le plaisir de la chair ${ }^{16}$, à s'affranchir d'un sentiment de victimisation et ce malgré les menaces qui pèsent sur leur mémoire. À travers ces différentes représentations, tout porte à croire que la quête de ce nouveau pouvoir résulterait du repli progressif de la censure qui est en proie à une mouvance féministe active et qui réclame un nouveau discours sur le corps et une légitimation de son existence.

C'est donc sans complaisance que les femmes, dans leur propre corps, s'assument complètement en réclamant une nouvelle posture et en souscrivant à cet idéal par le biais de l'écriture qui constitue le moyen approprié pour y parvenir. Cette écriture rebelle a, somme toute, réussi à repenser les vibrations du corps féminin en les disséminant dans le texte, en transcrivant l'écho de sa voix ${ }^{17}$, en fusionnant le verbe et la chair, l'être et le paraître,

en couverture des images portant sur le nu féminin renvoie brutalement aussi au corps du sujet regardant et interpelle son « pathos ». C'est le cas par exemple du paratexte pictural de Fracture du désir, qui reproduit la célèbre toile de Modigliani intitulée : Nu couché au coussin blanc.

14. Des micro-récits érotiques ponctuent souvent les romans de plusieurs autres romancières marocaines comme en témoignent les textes de Bahaa Trabelsi dans Une vie $\grave{a}$ trois (Casablanca, Eddif, 2000) et de Nedjma, dans L'Amande (Paris, Plon, 2004).

15. C'est dans ce sens que Michel Foucault considère que « c'est par le sexe en effet, point imaginaire fixé par le dispositif de sexualité, que chacun doit passer pour avoir accès à sa propre intelligibilité (puisqu'il est à la fois l'élément caché et le principe producteur de sens), à la totalité de son corps [...], à son identité » (Histoire de la sexualité 1 : La volonté de savoir, Paris, Gallimard, 1976, p. 205).

16. Pour essayer de comprendre le rapport de la chair au sens, Julia Kristeva soutient que la chair est « la complétude de cette incomplétude que m'impose la sensation. Moins qu'un désir qui a un objet, plus qu'une jouissance qui l'a perdu dans la fusion avec l'Être, la chair-chiasme entre sentant et sensible se tient aux exquises limites du senti. Ma chair ou la chair du monde? Tout comme. Imbibé de sens, mais reculant jusqu'à l'insensé : jamais conscience n'a été si audacieuse, et pour cela même si poreuse, que dans cette saisie de ce qui, en devenant moi, me déborde; [...]. Le monde me touche et je le touche - tu me vois et je te vois, co-présents et abandonnés. Une chair sensible recueille le temps sensible dans la communion des séparés. Massive et glorieuse à la fois » (Julia Kristeva, Le Temps sensible. Proust et l'expérience littéraire, Paris, Gallimard, 1994, p. 335).

17. Alfonso de Toro note dans son étude sur Nicole Brossard et Abdelkebir Khatibi ( Corps-Écriture ou écrire comme la circulation infinie du désir », dans Susanne Gehrmann et Claudia Gronemann (dir.), Les enjeux de l'autobiographique dans les littératures de langue française : Du genre à l'espace. L'autobiographie postcoloniale. L'hybridité, Paris, 
non seulement vers une altérité mais aussi et surtout vers une relation privilégiée de soi à soi. Ce genre de relation atteint son paroxysme, par ailleurs, chez d'autres écrivains masculins qui versent dans la reproduction d'un autre type de corps : homosexuel.

\section{Le corps de l'homosexuel}

Dans d'autres séquences narratives, la dimension sensuelle et érotique du corps, tant féminin que masculin, prend des proportions extrêmes au point d'occasionner l'apparition d' « héroïnes » ou de « héros » reconnus par leurs statuts de prostituée ${ }^{18}$ ou d'homosexuel. L'énonciation homosexuelle du corps est percutante, elle se manifeste particulièrement dans les écrits de Rachid O. ${ }^{19}$, de Mahi Binebine ${ }^{20}$ et surtout de Abdellah Taïa auquel nous empruntons ce passage, extrait de Le Rouge de tarbouche :

Je posais le miroir sur le lit étroit d'Abdelkébir dans lequel j'ai dormi tant de fois... avec lui (des heures longues et inoubliables, nos corps collés et nos odeurs mêlées). Je me rapprochais de la glace et je m'y découvrais. Le visage long, maigre : des boutons sur le front et le menton (j'adorais les faire éclater), des petits poils énervants dans le nez, l'œil droit légèrement différent de l'œil gauche, les joues creuses et affamées. Aucun charme. Oui, je suis comme ils ne disent pas, laid, inintéressant. Je m'abandonnais à ce narcissisme à la fois délicieux et douloureux. Je m'éloignais un peu du miroir pour

L'Harmattan, 2006, p. 67-101) que « le but de la voix du corps, de la corporalité et de la voix, ce n'est plus la transmission de messages ou d'émotions, c'est plutôt le déclenchement des "incidents pulsionnels". Nous pouvons le voir avec le langage tapissé de peau, par lequel peut être entendue la volupté des voyelles ou plutôt la stéréophonie de la chair profonde ». 18. Le corps de la prostituée s'empare de plus en plus de l'imaginaire romanesque et émerge à tous les niveaux du discours. Quelques exemples au choix sont à découvrir dans les œuvres suivantes : Demoiselles de Numidie et Au bonheur des limbes de Mohamed Leftah, dans le roman de Dounia Charaf, Fatoum, la prostituée et le saint ou encore dans la première nouvelle de Fracture du Désir de Rajae Benchemsi.

19. Salim Jay dans son Dictionnaire des écrivains marocains considère que « Rachid O. est le plus gidien des écrivains marocains. On aborde l'existence comme le terrain de la conquête et de la consommation homosexuelle. [...] Le ton unique de Rachid O. où s'affirme toujours une générosité du regard, triomphant de la mesquinerie, des sarcasmes et de la caricature » (Casablanca, Eddif, 2005, p. 299). Pour une analyse de l'écriture homoérotique, voir Khalid Zekri, Fictions du réel. Écriture du réel et modernité romanesque au Maroc, Paris, L'harmattan, 2006, p. 173-197.

20. Dans Le Griot de Marrakech de Mahi Binebine (Casablanca, Le Fennec, 2005), et plus particulièrement dans la nouvelle « Le baiser aux groseilles », le narrateur révèle qu'il se passionne au « jeu» de l'homosexualité avec son compagnon nommé Prospère Bocara. Extrait : « De retour [du cinéma] à notre refuge sur la terrasse, Prospère et moi prenions notre revanche en emboîtant le pas aux artistes. Nous repensions les scènes à la maison. Des fois j'étais l'héroïne et moi son amoureux, des fois l'inverse. [...] je me jetais sur lui et le serrai très fort dans mes bras, [...]. Nous nous embrassions tendrement [...]. C'était un jeu, le nôtre. Lascif et dangereux et que nous répétions à l'envi » (p. 56). 


\begin{abstract}
découvrir tout le reste de mon corps que je ne connaissais pas vraiment bien au début de ces retraites particulières. Encore oui, c'est maigre comme les chats des rues; et puis non, je ne suis pas maigre, je suis mince, c'est mieux quand c'est dit ainsi. Ma peau : à part celle qui recouvre mes mains, mes pieds et mon visage, je ne la connaissais pas non plus. J'enlevais mes vêtements pour la toucher (je passais mes mains sur le ventre, le torse, les seins, la nuque, les cuisses, les fesses, le sexe), la saluer, l'embrasser, la goûter ${ }^{21}$.
\end{abstract}

Dans ce passage, le miroir ne se réduit pas seulement à un objet qui sert à refléter la peau intime du protagoniste, mais il est aussi le moyen d'opérer une fixation sur sa masculinité. C'est en effet sous ses yeux que le corps en éclosion du protagoniste refait surface, peu à peu, et instaure, chez lui comme chez le lecteur, un embarras certain. Lors de cette mise en scène de sa propre nudité, l'acte de voir se meut en une perception fantasmée du sujet sur son propre corps en train de se dévêtir. Certes, si le miroir dévoile ici la nudité de l'homme, force est de constater, toutefois, que ce dernier est constamment en butte à sa propre vérité dont la découverte cristallise une relation de soi à soi nettement controversée. De la méconnaissance à la reconnaissance de son corps démembré, tous les vecteurs sensoriels (le regard, l'ouïe, l'odorat et le toucher) ont été investis pour lever le voile sur une peau ${ }^{22}$ qui se dérobait aux sens et dont l'appropriation devient voluptueuse et intense. Le désir du sujet de se mettre en scène suivant ce jeu de miroir se répercute instantanément sur l'acte d'écrire, acte selon lequel le « je » se voit en train de se réitérer inlassablement une douzaine de fois comme pour s'affranchir du regard stéréotypé d'autrui et dessiner une image de soi prompte et raffinée.

La matérialité transparente du corps de l'homosexuel est révélatrice ici d'un sujet narcissique, amoureux de son propre corps et de sa beauté. Les traits discrets de cette beauté commencent à se dessiner progressivement dans une communauté sociale qui réclame de plus en plus une image efféminée, heureuse et idyllique du corps masculin. Le culte de cette image relèverait, nous semble-t-il, d'une culture de l'apparence, de l'ostentation et du plaisir qui en découle. Il vise à découvrir chez le sujet homosexuel des manières de dire et de faire qui correspondent aux canons préalables voire stéréotypés d'un langage et d'une communication homosexuelles. Pour le lecteur, le sujet homosexuel a ceci de particulier qu'il porte les signes d'une diffraction inédite dans son identité qui s'emploie à réconcilier sa nature virile et son caractère efféminé. Face à cette corporéité hybride, il ne s'agit pas de l'exaltation d'une vision androgynique du corps; il ne s'agit pas non plus d'une « guerre de sexe », mais seulement d'un véritable effet

21. Abdallah Taïa, Le Rouge du tarbouche, Casablanca, Tarik, 2005, p. 23.

22. Mireille Calle-Gruber, dans une réflexion sur l'écriture de Assia Djebar, affirme que « la parole identitaire s'écrit toujours sur la peau » (cité par Katarina Melic, «L'exil et/ou la recherche d'une langue littéraire : Assia Djebar ou le blanc de l'écriture », Mots pluriels, $\mathrm{n}^{\circ} 17$ [En ligne] http://motspluriels.arts.uwa.edu.au/MP1701km.html\#fnB1). 
de mutation, d'incertitude et de perplexité. Les signes de cette mutation traduisent chez le sujet un double désir, celui de déconstruire son propre corps au moment même où il s'évertue à le reconstruire différemment. Ces mêmes signes seraient, en fin de compte, annonciateurs d'un imaginaire d'un corps « inimaginé » jusqu'alors dans la littérature marocaine contemporaine et qui s'apparenterait à une vision paroxystique du soi qui déculpabilise le sujet et le réconforte dans sa déviance même.

Le troisième type de corps qui est omniprésent dans l'imaginaire littéraire marocain et qui participe de cette variation qui nous occupe dans ce travail, concerne la figure du père. Le roman de Abdelhak Serhane, Le Deuil des chiens $^{23}$, cristallise un cas de figure extrême qui ébranle sérieusement la symbolique d'un corps considéré comme éminemment vénéré.

\section{Devant le cadavre du père}

Abdelhak Serhane s'applique, toujours, dans sa pratique littéraire, à joindre à l'acte de l'énonciation romanesque une dénonciation horripilante du corps paternel. En effet, dans le récit de Le Deuil des chiens, la figure du père est promise à la mort au moment où celle des sujets féminins, narratrices et personnages à la fois, est réhabilitée. Le sentiment d'une aversion poignante, d'abomination et de répulsion à l'égard de la dépouille du père, confère au discours féminin qui le prend en charge une autorité dont le seul pouvoir est celui du langage, de la loi du verbe. Â partir de cette loi du père qui était à l'origine même de la diégèse romanesque, le lecteur voit instituer dans le texte une autorité féminine. Celle-ci est habitée par le désir d'asservir et de subvertir en même temps le statut du père comme figure tyrannique par excellence. Ainsi, le « sexe faible » se voit attribuer le pouvoir d'agir et de dominer. La forme de cette domination inscrit la femme dans une relation perverse et rebelle, relation qui permet aux filles narratrices de rendre au père sa hargne naturelle qui fut la sienne :

On m'avait toujours appris à me taire, à écouter, à n'émettre aucun jugement, à ne faire aucune réflexion. Ma parole n'avait aucune importance. En parlant aujourd'hui, j'extorquais aux hommes et aux adultes un peu de leur pouvoir. Je faisais mienne une parole qu'on m'avait toujours confisquée. J'existais à travers ces mots de la nuit, même si mon récit n'avait aucune cohérence $^{24}$.

Si l'acte réprobateur a toujours quelque chose de masculin et de viril, il n'en est strictement rien dans le contexte du présent roman. Ici, le sujet tranche les interdits et échappe à toute instance morale en rapport avec

23. Dans cette partie, nous nous limiterons à l'étude d'un seul roman de Abdelhak Serhane, où le corps du père, réduit à sa dimension cadavérique, imprime tous les niveaux du discours romanesque : Le Deuil des chiens, Paris, Seuil, 1998.

24. Ibid., p. 170. 
le contexte de la formation culturelle du lecteur, de son éducation sentimentale, sociale ou religieuse. Le geste profanateur (l'acte d'exclusion des filles qui était à l'origine de leur déchéance) est fondé sur une perception culpabilisante du père. Cette perception se construit dans le texte en procès « judiciaire » avec ses plaidoiries, ses justifications, ses culpabilisations et ses sanctions.

Face à ce procès, les quatre protagonistes narratrices visent à reconquérir leur être et s'imposer comme sujets à part entière. Leur discours n'est donc pas complaisant, il est en revanche péremptoire et irrévocable. Le discours masculin cède ici la place à son substitut collectif, quatre voix présentes contre une manquante, celle du père absent. Cette promotion de la voix féminine rompt, sans doute, avec une doxa marquée essentiellement par le paradigme clos de valeurs masculines. Il ne s'agit pas seulement de relever cette opposition masculin/féminin, mais féminin/féminin : les quatre jeunes filles contre l'amante de leur père.

Ces dernières se réjouissent d'outrager la mémoire de leur père, dans une lutte contre le temps. En jetant l'opprobre sur le père, la narratrice souhaite en effet gagner la caution du lecteur. Le sujet refuse de se taire, il revendique le pouvoir de désigner l'autre en exposant son propre langage dans un jeu d'intériorisation/extériorisation, dans un mouvement d'énonciation/dénonciation. Le texte dit de vive voix ce que le lecteur ne doit pas entendre et met en lumière ce qu'il ne doit pas voir comme en témoignent ces exemples :

Tu es mort aujourd'hui, mais la mort est incapable de te protéger contre la violence de notre parole et de notre haine ${ }^{25}$.

Rien ni personne ne peut m'empêcher de suivre ton cercueil jusqu'au cimetière, rien que pour rendre ton enterrement illicite puisque les femmes n'ont pas le droit d'accompagner un mort lors de ses funérailles ${ }^{26}$.

Le cadavre devait nous maudire mille fois pour l'épreuve que nous lui faisons subir [...]. Je renonçai au spectacle du mur, oubliai la présence de mes sœurs et l'odeur prenante de la mort, $[\ldots]^{27}$.

Lorsque le corps paternel est fortement calomnié, lorsqu'il est emprisonné dans sa dépouille mortelle, réduite strictement à sa chair en décomposition, tout semble donc devenir insoutenable et ineffable à la fois. Dans ce contexte pernicieux et morbide, le plaisir incisif du corps de la narratrice de « toucher » le corps paternel anéanti, conférerait au récit une dimension quasi « anthropogénique » qui offense le lecteur dans ses schémas habituels d'accomplir le deuil et de le penser. Car non seulement le corps paternel est révélé dans son cadavre, dans sa nudité et sa vulnérabilité face aux attaques

25. Ibid., p. 266.

26. Ibid.

27. Ibid., p. 182-183. 
des insectes qui le ravagent mais aussi aux mots irrépressibles des jeunes filles qui percent sa chair comme pour lancer un défi à la mort même. Le corps du père est poursuivi au-delà de sa limite, à l'extrémité de son être.

Par ailleurs, si le texte de A. Serhane est porté par une énonciation et une subjectivité intenses, est-ce pour infliger un affront à la condition du corps humain ou est-ce pour cristalliser un lieu névralgique de la catharsis? À travers cette image du corps en décomposition, c'est, nous semble-t-il, toute la vision du réel qui est démantelée et ébranlée. Et la narratrice semble réinventer le réel sur fond de ses propres désirs et de ses propres phobies. Le désir de s'exprimer devant un corps absent excite davantage la narratrice à se livrer avec un plaisir inavoué à aller jusqu'au bout de la revanche, en affûtant un discours de l'obscène, du cruel, et ce jusque dans ses excès. Renonçant à se taire devant des mots censurés, la narratrice, dans les passages suivants, s'obstine à s'en délecter comme pour déjouer la censure :

J'avais l'oreille fine et j'étais capable de distinguer le frémissement d'une étreinte érotique, le frôlement d'une robe qu'on soulève, la caresse imperceptible d'un sein ou d'un entrecuisse [...] même dans la nuit et à distance, je pouvais percevoir le froissement des chairs enlacées dans une lutte amoureuse $[\ldots]^{28}$.

[...], Tamou se redressa, enjamba le cadavre et se dirigea vers la porte ${ }^{29}$.

Ainsi, de la simple description de la chair physique du corps, le texte passe pour devenir le lieu d'une dénonciation de la métaphysique. De l'instant de l'exposition du corps à son ensevelissement, naît le livre de Serhane. Le romancier prend à rebours cette figure culturelle du masculin, figure d'un père mort, se trouvant en dehors de l'histoire, et qui s'instaure sur un seuil et une limite de l'énonciation et de la nomination.

\section{Le corps intersémiotique}

Dans un tout autre registre de nature essentiellement intersémiotique, le corps est doté de nouvelles fonctions esthétiques. En parcourant le dispositif plastique à l'œuvre dans la littérature marocaine contemporaine, le corps revient souvent dans des positions poétiques et plastiques, au niveau du paratexte et dans le corps même du livre. Démembré ou unifié, nu ou vêtu, sa figuration plastique renseigne aussi sur la position éthique ou axiologique du peintre.

Dans un livre exemplaire d'Abdellatif Laâbi ${ }^{30}$, les images de Kacimi qui accompagnent les fragments poétiques, représentent le corps comme

28. Ibid., p. 94.

29. Ibid., p. 263.

30. Ruses de vivant, illustrations Mohammed Kacimi, Neuilly-sur-Seine, Al Manar, coll. « Poésie du Maghreb », 2004. Ce genre de livre picto-scriptural, appelé par ailleurs 
une construction chaotique de lignes exsangues arrachées à une surface où s'enchevêtrent les traits et les traces d'une identité défigurée et sans couleurs, au visage assombri, sans chair et sans lumière. Les dessins dépouillés de Kacimi ${ }^{31}$ inquiètent en effet le regard du lecteur. Des lignes enchevêtrées, tendues et distendues, se posent comme les traces d'une mémoire et des fragments qui, témoignant d'un vécu, se refusent au discours et à ses lois. Ces lignes aux formes convulsives qui entourent le corps crayonné ou l'encadrent, brouillent la perception du sujet et le confinent dans un sentiment de désarroi et d'apitoiement. La projection de ces lignes éclatées et ondulatoires est aussi symptomatique d'un embrasement intérieur qui ronge l'esprit du sujet peignant et contourne son corps. Aux yeux du lecteur, l'image ${ }^{32}$ du corps dessinée dans cette posture, mutilée et meurtrie, traduit une expérience au seuil du tragique et de la mort. Pour Laâbi, comme pour Kacimi, dessiner la mort ou la dépeindre, tel semble être la ruse ultime du poète qui voit son double franchir irréversiblement « le mur de la lumière ${ }^{33}$ ». Cet ultime voyage lugubre trouve en effet son écho dans la parole poétique comme on peut le lire dans les vers suivants :

\author{
Ce bout de route \\ devant moi \\ plus proche de la nuit \\ la vraie \\ la véridique \\ l'incontournable \\ Je ralentis le pas
}

\begin{abstract}
« illustré » ou encore « d'artiste », incarne-t-il une véritable osmose entre texte et image ? ou obéit-il à une scénographie éditoriale qui ne dit pas son nom? Le lecteur donnera-t-il plus d'importance au texte sous prétexte de représenter un lieu de l'ekphrasis et donc d'aider à la compréhension de l'image? ou à l'image sous prétexte d'illustrer le texte dans une autre tradition classique de l'ut pictura poesis? Cette interaction de ces deux médiums, littéraire et artistique, est la source d'une forme d'hybridité dont les enjeux esthétiques et éthiques imposeront aux auteurs et aux lecteurs un mode particulier de production et de réception. D'autant plus que les fonctions assignées au dispositif plastique ne sont plus illustratives ou ornementales mais heuristiques et esthétiques. Notons aussi que la conception de ce genre de livre est en soi problématique, voire subversive, car il semble qu'un travail préalable de tri et de choix se soit opéré pour faire correspondre telle image à tel texte, telle disposition à tel support, tel genre à telle image. D'où la facticité inhérente à la mise en page et à la mise en image du livre. L'émergence de ce dernier dans le paysage littéraire et culturel au Maroc semble donc rompre avec la tradition du livre classique, sa pratique éditoriale, ses circuits habituels en imposant de nouvelles configurations typographiques, formelles et iconiques. 31. Mohamed Kacimi : «Le dénominateur commun à toutes mes toiles est l'inquiétude du sens » (Parole nomade. L'expérience d'un peintre, Approches et Rencontres, Neuilly-surSeine, Al Manar, 1999, p. 32).
\end{abstract}

32. Nous nous contentons de reproduire, en fin de cette partie, deux dessins de Mohamed Kacimi que Laabi a incorporés à son livre, Ruses du vivant, Neuilly-sur-Seine, Al Manar, coll. « Poésie du Magrhreb », 2004.

33. Abdellatif Laâbi, Ruses de vivant, op. cit., p. 7. 
Je fais semblant

d'admirer le paysage

Ruse de vivant ${ }^{34}$.

Tout semble ainsi transiter par le corps du peintre et du poète. La souffrance de la mémoire, le deuil du présent et la mort à venir. Ruser avec la mort c'est aussi accomplir son deuil poétique. Ainsi :

\author{
Quitter \\ ne sera pas \\ la première déchirure \\ ni le premier scandale \\ Est-ce vraiment \\ l'ultime exil ${ }^{35}$.
}

Devant l'image, pour reprendre ici le titre d'un essai majeur de Georges Didi-Huberman (Paris, Minuit, 1990), la parole poétique, chez Laâbi, est lapidaire. Elle émerge du fond blanc de la page comme pour briser le silence envahissant de la toile et de l'écriture ou pour retourner au secret du silence intime qui les fait naître. Les mots de la poésie se font rares, limpides et hermétiques à la fois.

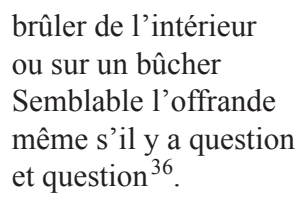

Les mots du poète sont emportés dans un flux verbal constant et immuable au point que le lecteur ignore s'il s'agit d'un seul poème ou de plusieurs. N'utilisant que des points d'interrogation, le lecteur est souvent apostrophé par un sujet qui s'interroge sur " les raisons ou les déraisons de son écriture et de $\mathrm{sa} \mathrm{vie}^{37} »$. Ce questionnement poétique trouve son écho dans la représentation plastique du corps dans la mesure où les dessins de Kacimi et le poème de Laâbi expriment dans un mouvement intersémiotique bien ordonné, un imaginaire « iconotextuel».

Dans cette perspective, le corps plastique et le corps poétique se reproduisent dans des positions existentielles qui oscillent entre l'espoir et le désespoir, la vie et la mort.

37. Abdellatif Laâbi, L'Écriture au tournant, texte accompagné par Mahi Binebine, Paris, Al Manar, coll. «Approches et Rencontres », 2000, p. 9. 

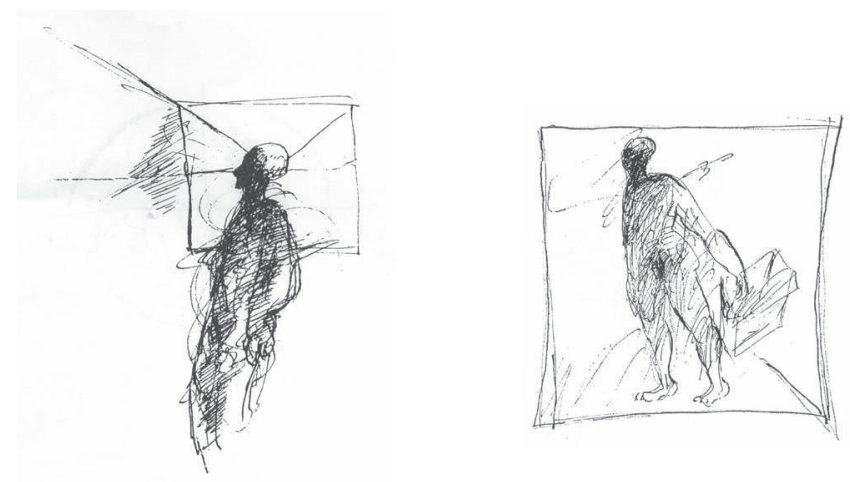

Dessins de Kacimi incorporé au livre de Laabi, Ruses de vivant, Neuilly-sur-Seine, Al Manar, coll. « Poésie du Maghreb », 2004. À gauche, p. 31, à droite, p. 27.

\section{Conclusion}

Au terme de cette étude, il apparaît que dans les œuvres de fictions que nous avons abordées, la représentation du corps prend des proportions de plus en plus considérables. Pris en charge par une pluralité de voix, féminines et masculines, elle contribue à l'érosion d'une conception implacable et rigoriste ancrée dans la tradition socioculturelle marocaine. Dans cet imaginaire littéraire, le corps se pose ainsi comme le lieu d'une médiation et d'une variation qui rend manifeste les fluctuations que subissent les sujets dans une société tiraillée entre la tradition et la modernité, la fascination pour le désir du corps et l'effroi qu'il suscite lorsqu'il devient vif, exalté et déchaîné. Depuis une vingtaine d'années, en effet, la littérature féminine tout particulièrement, s'est servie du corps comme fondement à son « souffle ", comme lieu d'un cri inaugural qui est toujours en cours d'accomplissement. Aujourd'hui encore, ce corps ne cesse d'effectuer son éternel retour, réinvesti et renouvelé à chaque fois et inlassablement. Ce renouvellement fait naître une énonciation corporelle imprégnée d'une subjectivité lancinante de l'être en quête d'une condition humaine décente et idyllique et d'une identité qui reste à construire.

Abdallah Lissigui

Faculté Polydisciplinaire de Taza Université Sidi Mohamed Ben Abdellah, Fès 